\title{
SUPPORTING RELAXATION: SLOWBREATH FOR PEOPLE WITH MYALGIC ENCEPHALOMYELITIS
}

\author{
Jorun Børsting \\ Department of Informatics, University of Oslo, Oslo, Norway
}

\begin{abstract}
This paper describes the design process resulting in a cushion for relaxation called SlowBreath. The cushion was made with the intent to support people with Myalgic Encephalomyelitis (ME) in illness self-management. In particular, the focus was on energy balancing (activities interlaced with relaxation and rest) that people with ME need to master. When SlowBreath was finished to the extent that it could be regarded as a research product, five people diagnosed with ME participated in exploring the fit of SlowBreath in their lives, as well as the experiences that it generated. In this paper, the importance of design for minimal interaction for this particular user group is highlighted. The findings from the study are articulated as a set of implications for the design of everyday digital artefacts, aiming to scaffold self-management for people with ME.
\end{abstract}

\section{KEYWORDS}

HCI, e-Health, Relaxation, Myalgic Encephalomyelitis, Self-Management, Research Products

\section{INTRODUCTION}

Myalgic Encephalomyelitis (ME) still has no scientifically established cause and subsequently, no curative treatment. It is established, however, that ME is a chronic, multisystem illness that severely affects the life quality of people diagnosed with the illness (Hvidberg et al., 2015). ME is classified in four primary categories, ranging from very severe (completely bedridden), to mild (reduced in function by at least 50\% over a long time) (Carruthers et al., 2011). The illness is also considered to be common, with studies indicating prevalence as high as 3-5\% by some definitions of the illness (Líndal et al., 2002). Post-Exertional Malaise (PEM) is a key symptom, present in all people with ME. A cluster of other symptoms is also usually present. The most frequent ones are hypersensitivity to external sensory inputs (touch, smell, taste, visual, or auditory), fatigue, sleep disturbance, muscular pain, nausea, stomachaches, headaches, immune dysfunction and impaired concentration and short-term memory. PEM causes intense exhaustion, and an increase in the intensity of other symptoms after mental or physical exertion. The exertion needed to trigger PEM is different from person to person and sometimes varies with the same person on a day to day basis. For some, it might be caused by a small amount of exercise. For others, everyday activities like doing the laundry, or sitting upright, can trigger the PEM. While struggling with these fluctuating symptoms and health issues, self-management strategies seem to be, at present, the best option. Energy balancing (Lacourt et al., 2018) is one commonly used self-management strategy which involves balancing periods of rest and activity to avoid overexertion (Goudsmit et al., 2012).

Only recently, has technology design for people with ME received attention within the HCI community (Culén et al., 2019). People with ME are found to present a particularly interesting technology design challenge, based on how they need to minimize their interactions, both with technology and other people. Furthermore, exploring how technology can support self-management strategies, such as energy balancing or increasing wellbeing, becomes relevant.

This paper explores whether relaxation, or meditation, facilitated by digital artefacts such as SlowBreath, see Figure 1, can be a component in energy balancing and self-management of ME. SlowBreath gently guides and supports users at moments when relaxation and de-stressing are important. The aim of the work presented in this paper, was not to establish practices of regular relaxation or meditation by using SlowBreath. Instead, the focus was on identifying design implications for digital artefacts that can scaffold 
experiences of relaxation and meditation, by exploring form, computational materials, the materiality of artefacts, and bodily experiences that they facilitate. In addition, the paper contributes by addressing methodological challenges of doing research for and with vulnerable people and proposing an alternative to traditional participatory approaches.

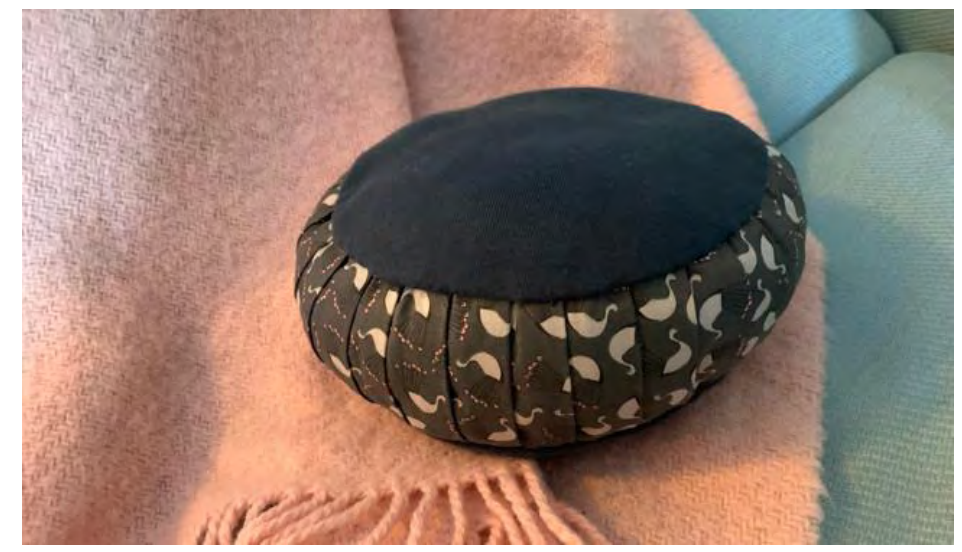

Figure 1. The designed artefact, SlowBreath, aims to scaffold relaxation and meditation practices

\section{RELATED WORK}

In this section, related work is divided into two main themes: technology design and $\mathrm{ME}$ and research on relaxation and meditation in HCI.

\subsection{Technology Design and ME}

As mentioned in the introduction, designing technologies for people with ME has only recently gained attention within the field of HCI. Culén et al., (2019) made the observation that aspects of rich communication and minimal interaction (Janlert and Stolterman, 2017; Kaye, 2006) are relevant for technology design for people with ME, who require minimal physical, cognitive, and sensory exertion. Furthermore, a framework consisting of a set of strategies for designing technologies that support relatedness (while involving minimal interactivity and engagements) was proposed. The strategies were physicalness, expressivity, awareness, balance, extension-of-self, coolness, and acts-of-care. The strategies were developed based on a study with a research product. In that study, the research product was a networked robot, placed in the everyday lives of nine adolescents with $\mathrm{ME}$ to explore the extent to which the robot mitigates the sense of social isolation. The findings indicated that the research product was highly effective at improving the social relatedness of participants, while adhering to the need to reduce interactivity and engagements to a minimum. Research products are to be understood as described by Odom et al. (2016), as artefacts having the interrelated attributes Inquiry-driven, Finish, Fit and Independent. Inquiry-driven implies that experiences with the artifact drive the research inquiry. The Finish highlights the actuality of the design artifact, i.e., what it is, rather than what it might become. The Fit relates to the lived experiences with the artifact. Notably, the artifact should be perceived as neither too strange nor too familiar, for the artifact to gain an actual role in the context of users' lives. Lastly, the Independent implies that a research product can be used in everyday life without interventions from the researcher.

The research products used in this study was instrumental for gaining better insights on how the product would actually be used and to what extent it would fulfill its intent. This is important in general, but in particular when the research involves vulnerable users. Similar ideas did also guide the present work. 


\subsection{Research on Meditation in HCI}

The rapid pace of modern life, has increased the interest in ancient practices of mindfulness and meditation as ways to cope with high levels of stress, thus improving the health and wellbeing. Here, the term mindful meditation is used to describe the process of taking time to slow down and focus on the present moment (Kabat-Zinn, 1990). Prior research on mindfulness meditation has shown that it can produce a variety of health benefits (Brown et al., 2015). For beginners in mindfulness meditation, breathing exercises are often the best way to start. Either human voice or meditative music guides the breathing. It is difficult for novice meditators to remain focused during meditation. Therefore, it frequently takes months of regular practice to develop a state of flow in the meditation (Cochrane et al., 2018).

Currently, in HCI there is a growing focus on mindfulness, wellbeing, and technology (Calvo and Peters, 2014; Niksirat et al., 2017). Several studies within HCI have investigated how to design technologies for mindfulness meditation to increase wellbeing. Most common approaches provide guided meditation through various senses like touch, audio, and vision (Niksirat et al., 2017). Several studies also focus on the user's bodily experiences (Bergström and Jonsson, 2016; Höök et al., 2016) as a guide for the design of meditation technologies. Others, provide visual feedback in real time, displaying bodily states based on data from wearable bio-sensors (like breathing or heartbeat) to encourage mindfulness and developing meditation strategies to increase wellbeing (Sanches et al., 2010).

Prpa et al., (2018) performed a research through design study focusing on breath and mindfulness meditation. They explored how people become aware of breath through embodied interaction, through the design of an immersive virtual environment with sounds to facilitate breath awareness as part of meditation. They found that participants' own accounts of their experience can describe their breathing data. Further, they state that their study: "revealed the process of becoming aware of breathing in VE and demonstrated the importance of a sense of agency, understanding of control and possible subtle impact of the environment on breathing patterns without users being aware of it" (ibid., p.80).

The somaesthetics, in general, focuses on research in HCI that engages with the body (Shusterman, 2012). Two particular examples are relevant to mention. The Soma Carpet and Breathing Light are research artefacts designed with a somaesthetic approach to meditative bodily introspection (Höök et al., 2016; Ståhl et al., 2016). The Soma Carpet provides heat stimuli to subtly guide the attention of people interacting with the artefact to different body parts. Furthermore, the Soma Carpet is described to support guided meditation, body scanning, Feldenkrais-sessions, or to facilitate relaxation (Ståhl et al., 2016). With a similar aim, The Breathing Light lamp creates an ambient light dimming connected to data from breath. The Breathing Light also uses sounds, like the sound of waves or bird songs, to facilitate the meditative experience. The studies revealed that the Soma Carpet and the Breathing Light are successful in generating a feeling of relaxation, softer movements, and increased awareness of breath (Ståhl et al., 2016).

This research aims to reduce interactions even further, focusing on the very subtle vibrational guidance of the breath and warmth towards relaxation.

\section{FRAMING THE RESEARCH}

In this study, research products were used to perform inquiries into how technology can support the self-management of ME. The inquiry was performed through material explorations in the everyday lives of five people with ME. Due to the nature of explorations, the research was qualitative.

\subsection{The Method}

The main method was in-depth interviews with participants. The author traveled to the homes of the participants for an interview, and left SlowBreath in participants' homes for at least a week. During this period, participants were to use SlowBreath freely and on their own terms. The elicitation diary was used to collect data on the use. The participants were given notebooks for the purpose. Whenever the participant was ready to return the artefact, the second visit, and the second interview took place. The involvement of the researcher in the field deployment was minimalized, to lower the strain of participating in the study for the ME ill participant, as described in (Culén et al., 2019). The data from the research product deployment was analyzed using content analysis with a-priory coding (Lazar, 2010). 


\subsection{Participants}

The five participants in the study all have mild to moderate ME diagnosis. Four participants are female, and one is male. In the rest of the paper, pseudonyms are used to preserve their privacy. Hedda and Mathea had a rather similar living situation, both live in an apartment on their own. Elin lives with her fiancé. Astrid lives with her husband and two children and has had the ME for fourteen years, through the largest part of her children's childhood. Henrik lives with his parents.

\subsection{Ethics and Dissemination}

A strong focus on research ethics and how to involve participants in the research while reducing negative health implications due to the energy needed to participate in the study was an important ongoing concern. The study was also reported to and approved by the national ethical review board.

\section{THE DESIGN OF SLOWBREATH}

The SlowBreath did not emerge through the ideation process, or in an instant. Rather, through four years of research with technology for people with $\mathrm{ME}$, the common concern with how to relax and know when to do it emerged as important. All people with ME who crossed my research trajectory struggled with energy balancing, and almost all had limited experience with meditation. Thus, the design focused gradually on making a device that gently supports users in relaxation or mindfulness meditation. Through the vibration, the user was given a guided breathing experience in time intervals lasting four-seconds. Both the duration of the breath and the quality of vibration could be, in theory, endlessly manipulated until the 'right' combination for a participant was found. However, two particular patterns were explored with participants. The first one was a four-seconds breath pattern, inspired by traditional mindfulness meditation practices. The second pattern was giving repetitive pulsing vibrations to increase mindfulness. The heat functionality was intended to be calming and relaxing. The combined use of heat and vibration aimed to give participants the 'ideal' combination to reach the relaxed, meditative state.

In line with (Mazé and Redström, 2005), this research explored a plethora of combinations and dynamics of the materials, forms, and computational expressions to create the SlowBreath, Figure 1. In what follows, excerpts from the initial interviews with users provide for a richer understanding of the condition. In the light of this, the choice of the shape, computational materials, and other materials are discussed.

\subsection{Energy Balancing and Stress}

When asked about what living with ME implies, Elin said: "People often don't understand that having ME is so much more than just being tired, the symptoms and PEM are so much more complex than just being tired". All participants discussed the PEM, and how it was triggered for them. They all wished they had an explanation (medical) for what it is that they have. Elin addresses the enigma of PEM: "A lot of effort goes into trying to find patterns that could give insights into where my limits are in regards to overuse of energy, or what it is that exactly makes me worse".

All participants had sleep difficulties, both in regards to poor sleep quality, and non-restorative sleep that affected their energy levels. Two of the participants had developed a strategy of looking at data about sleep quality in the morning, as one of the indicators of their energy level on a particular day. Hedda got the sleep quality data from her Fitbit, while Astrid got it from her CPAP machine, that she uses for her sleep apnea.

However, self-monitoring the illness through logging and self-reporting can be difficult. Elin pointed out that she was on a self-management course for the people with ME, where she was to log her energy levels extensively. The tracking was not working for her: "The tracking was misplaced, since I can't trust my feelings at the moment as indicators of where my limit goes. Because if something is too much, I do not feel that at the moment. I can feel good doing something, but the next day I find out that it was all too much what I did the day before". She further explains: "It feels very varying what I can do and not do. I can do 
something one day and endure it fine, but the next day I do the same thing and get really sick". In addition, the act of tracking extensively was making her sicker.

The participants describe the enigma connected to the cause of PEM as a huge challenge, especially since it affects their social lives. When their condition is so unpredictable, it makes planning (desired) social activities difficult. All participants admitted that they sometimes do activities that they know will cause PEM, but they went ahead because they felt like doing something important or meaningful, no matter the cost. However, for all participants, such overuse of energy resulted in weeks or even months of having increased illness symptoms and often being entirely bedbound during that time.

Hedda draws attention to another common problem of self-blame: "Everybody has their window of opportunity where there are small things that they can do to affect their daily health. However, it is also a part of the illness to get really sick without having done anything wrong in regards to self-management and energy balancing. Sometimes, the illness simply gets worse. It is important at that time to remember that if you get worse and worse, you should not blame yourself. What we know for sure, is that energy balancing can often help people with ME. It comes without any risks of worsened health for the individual. Since there is no cure for the illness yet, energy balancing is the only 'treatment' we have now that can have an effect". Henrik addresses his concern regarding the increased focus on energy balancing: "I can become scared of over-exertion and what happens in my body if I go beyond my limit. It is very important to focus on energy balancing, but not to the point where it becomes scary to do anything". He further elaborated that being scared resulted in a feeling of being stressed and provided an example of how technology for self-management of ME can be stressful: "If you are, for example, going to have something to remind you to rest, it must provide reminders in a way that doesn't make you stressed".

From all of the above, it is difficult for people with ME to not feel the stress of living with the condition. In fact, the states of anxiety, confusion, even panic are frequent. Ways to relax then can be a part of those small daily things that can be done by ways of self-help.

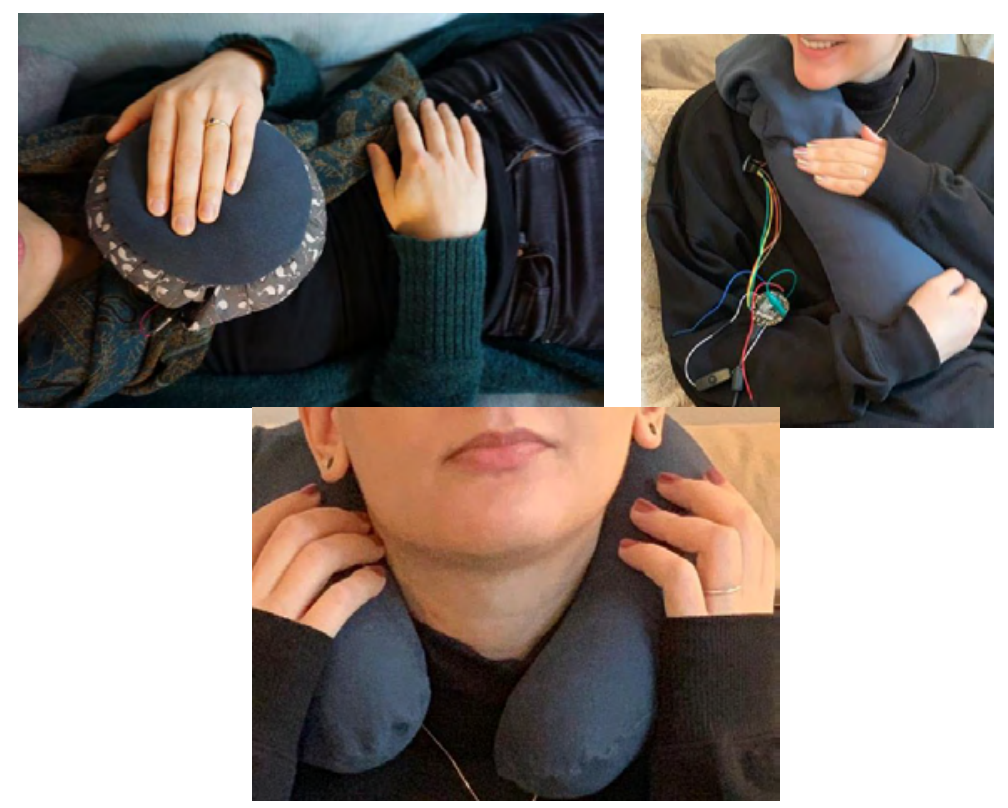

Figure 2. (left) How Hedda used the circular shaped SlowBreath for the first time. (middle) Elin trying the rectangle shaped meditation cushion. (right) Elin trying the U-shaped cushion

\subsection{Three Shapes to Explore Bodily Experiences of Participants}

As mentioned, there were developed three distinct shapes, in the following, the forms are presented, in addition to the selected computation and materials. Also, an account is given of how participants interacting with them during interviews perceived these shapes. The three distinct shapes were used to explore materials, forms, and shapes through bodily experiences of artefacts similar to soma shapes and tools (Höök, 2018). 


\subsubsection{Form}

Several shapes and sizes were used (see Figure 2) and addressed during interviews with the participants to explore how the form shaped the properties of the meditation artefact. There were three main shapes:

i. $\quad$ Long and rectangle shaped, it was weight down by its filling that was buckwheat.

ii. U-shaped cushion, very lightweight filled with small Styrofoam balls.

iii. Circular shape filled with poly-fil.

All the different meditation cushions had reused fillings, and the outer material was ecological cotton. While all three shapes had different qualities that in some way were relevant for generating meditative experiences, the circular shape was found to be more configurable during the design process, and was thus selected to be used as research products placed in the everyday lives of participants for a little over a week of use. However, the other shapes and different size variations of the circular shape was used during interviews, to further inquire into the bodily experiences of all the developed shapes.

\subsubsection{Computation}

SlowBreath was programmed in Arduino and Python, it contains an Adafruit FLORA microcontroller, two electric heating pads, five vibrating mini discs, and one haptic motor driver. Through changing the code controlling the vibration as part of the material exploration, it opens up for investigating how the temporal form and additional properties of SlowBreath were manifested through interacting and using the artefact.

\subsubsection{Material}

SlowBreath was sewn of ecologic cotton fabric and filled with repurposed poly-fil. Through the exploration of materials bringing the fabric closer to something natural by choosing cotton over synthetic materials became important. The material was a key aspect of shaping the feeling of holding and touching SlowBreath and experiencing the vibration and heat provided by the computation.

\section{FINDINGS}

The findings are presented from the bodily experiences with the three different shapes and lived experiences with the research product.

\subsection{The Bodily Experiences of the Three Shapes}

In this section, the findings regarding participants perceptions of the three shapes are provided.
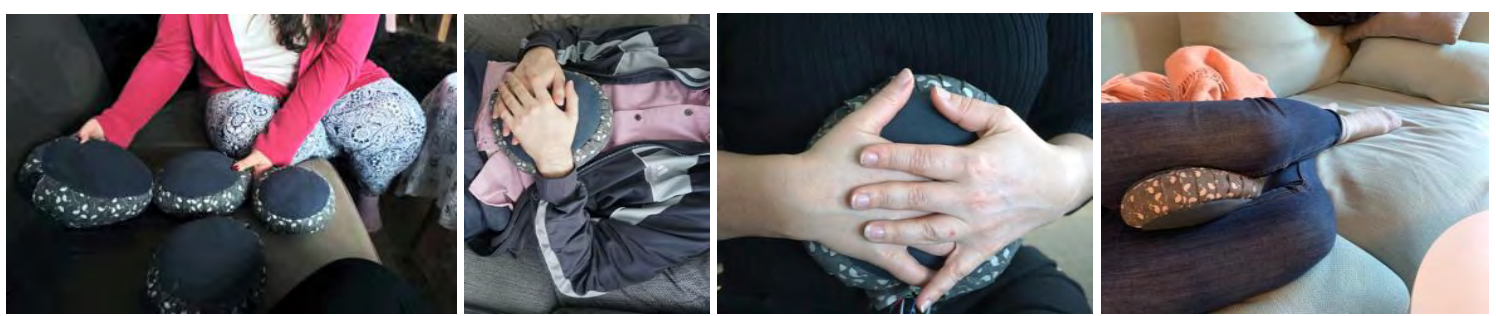

Figure 3. (left) Mathea exploring different sizes of SlowBreath, (middle left) Henrik trying the guided breathing, (middle right) Astrid using the meditation vibration functionality (right) Hedda using the heating functionality

\subsubsection{Rectangle Shaped Meditation Cushion}

The rectangle shape reminded the participants of the heat pads that can be heated in the microwave oven pointing out that the cushion had similar shape and weight. All participants liked the weight of the rectangle shaped cushion. Elin commented: "I like the feeling of the added weight given by this cushion. For me, the weight is calming". Hedda similarly commented: "I like the heaviness. For example, I also think that a large, heavy duvet is more relaxing than a thin blanket when sleeping. With the added weight, it provides conditions for relaxation in a good way". 


\subsubsection{U-shaped Meditation Cushion}

The u-shaped cushion gave clear associations to traditional neck cushions, which also restricted the participants imagined ways of using this meditation cushion. Elin addressed this by stating: "I cannot see myself using it in other areas than the neck, I would feel quite stupid by embracing or cuddling something that looks like a neck cushion. The other cushions can be used in many different ways more naturally than the neck cushion one". However, the participants did like to use the heat functionality at their neck area, as it was described as a body part that was often cold.

\subsubsection{The Circular Shape of SlowBreath}

The notion that the circular shape is more versatile surfaced during the research explorations in the design process and was validated through interviews with participants. Elin's initial comment regarding SlowBreath was: "It is so lightweight and can be versatile in use". She immediately placed SlowBreath at her chest, with the heat towards her body and placed her hand on the vibration. She stated: "It feels good to have the cushion on my chest and the added heat is great". She also commented that the meditation cushion would be suitable for placing on her abdomen to visually follow her breathing during the meditation. When first introduced to SlowBreath, both Hedda and Astrid laid down and placed it on their chests. In contrast, Henrik and Mathea both placed it on their abdomen. However, all participants were actively exploring different placements of SlowBreath during the first interview.

Scaling up and down the size of the cushion was also explored. A total of five different sizes of SlowBreath were made, as can be seen in Figure 3 (left). The participants interacted with the different sizes of cushions. It became evident that perceived affordances of the artefacts varied based on size. Hedda and Elin perceived the smallest cushion as toy-like, as Elin articulated: "the smallest meditation cushion feels like a kind of teddy-bear. Intuitively, I just want to lift it and cuddle it." The largest meditation cushion was seen as an ordinary cushion that invited to be used as a head rest, or placed in-between one's legs for added comfort (relevant for those who are in laying down position a lot). However, the sizes in-between these two were found to be more ambiguous as Hedda elaborated: "I think it loses its teddy-bear feeling already at one size bigger. The smallest is fun and cute, the biggest is clearly a cushion. But the ones in the middle are a little unrecognizable. They are a mysterious thing that one does not know what is". The ambiguous aspect of the middle-sized cushions also sparked curiosity, as Astrid pointed to: "I kind of like the middle-sized version, somehow it makes me curious about what it can do for me, and I like that feeling".

\subsection{Lived Experiences with the Research Product}

The mid-size, circular shaped cushion was used in the field study. The aim of the study was to allow the participants to experience SlowBreath in their everyday lives and for relaxation, as part of energy balancing. The closing interview with participants contained rich descriptions about their individual, lived experiences with the cushion. The analysis of interviews focused on the a-priory categories: 1) bodily experiences 2) relevance of the cushion for energy balancing, 3) tension between meditation and relaxation, and 4) feedback on the design with suggestions for re-design, which are used bellow to organize the findings.

\subsubsection{The Bodily Experiences}

The vibrational functionality was liked by all participants. Some needed to get used to it, as Elin describes: "The vibration functionality to guide the breathing was comfortable, but it took some time to get into it. I like it”. After testing SlowBreath in their homes, Elin and Hedda, as first users of the cushion, did raise some issues. For Hedda, the mindfulness breathing was new, and she found it challenging. She would have liked to keep the cushion and try more, but with different vibration patterns. She described her difficulty: "I tried to breathe with the vibration, but I got so out of breath and just wanted to breathe normally. So, it was hard and tiring to follow the guided breathing". Elin found the vibration and the heat to be too intense for her to facilitate a relaxing and meditative experience. Even though Hedda and Elin did not have positive experiences with the implemented functionality, both expressed that the potential of the cushion is great and that with lower intensity of the vibration and heat it could have been quite beneficial toward relaxation. The vibration and the heat then were adjusted, impacting very positively the experiences with the artefact for the 
remaining three participants. This also showed how even minor alterations to the computation materials had a significant impact on bodily experiences.

Astrid's experience with SlowBreath was very positive: "I have used the guided breathing the most, it was easier to find a rhythm in my breath with it. I experienced that the four seconds vibrations calmed down my breathing also. I usually find it difficult to calm my thoughts, but this too was easier with SlowBreath. I could relax every time I used it."

"It felt very comfortable to just lie down and feel the vibration", Mathea pointed out, elaborating on her experience of using SlowBreath: "When using SlowBreath, I noticed that I draw in the breath with the first vibration and then at the moment when I start breathing out the next vibration comes naturally. I experienced that I developed a nice rhythm in tune with SlowBreath, the intervals felt really nice. And that experience shows how the name [SlowBreath] reflects exactly what it does".

Henrik described his experiences with SlowBreath as positive - on most days. On days when the illness symptoms were increased, the vibration was still perceived as too strong.

\subsubsection{Potential of Being Incorporated in Practices of Energy Balancing}

Hedda, although she thought that the cushion could be very useful, had some reservations for her own use. She had been sick for so long that she had developed her own practices of resting as part of energy balancing. Thus, she was not sure if she would be incorporating SlowBreath in her life as a regular practice of relaxing.

Henrik's conclusion was similar: "Since I've been sick for a while, to go and rest is so natural for me. I somehow know exactly what to do, and how to control my breathing. If I would now start using SlowBreath, it will be a bit distracting. But then, if I had SlowBreath earlier in the course of my illness, it would have been ideal." He also pointed out that he still finds it challenging to relax through breathing exercises when he is not at home, like when traveling, or sitting at the doctor's waiting room. He saw the potential for himself to use SlowBreath in such situations.

Mathea, Elin, and Astrid had not resolved challenges related to relaxation practices, bringing forward the fact that they are very easily distracted. Mathea found SlowBreath successful in helping her keep the focus on the breath, thus, leading to less distraction. She would have liked to incorporate SlowBreath in her current practices of energy balancing. Also, Astrid reported that SlowBreath was very successful in helping her meditate and experience fewer distractions, making her regular practice easier and better.

All participants meant that SlowBreath had a potential to help develop practices of relaxing, and energy balancing, in the early onset of the ME illness. Using SlowBreath in the course of this study, made all participants reflect on how their own practices of energy balancing evolved since the onset of the illness.

\subsubsection{Resting vs. Mindfulness Meditation}

Prior to the engagement in this study, an inquiry on meditation was done with the ME organization. It revealed that there are two fractions: people who are positive towards meditation, and the ones that are negative. Hedda addressed this directly: "I know that the ME community is very divided when it comes to meditation, about half practice meditation and finds it helpful, while the rest prefer to rest without meditation".

The participants in the study also had different personal opinions about meditation, especially prior to using SlowBreath. Elin, Astrid, and Henrik like meditation, while Hedda and Mathea do not find mediation useful and were critical towards its role in energy balancing. Mathea had a strong reaction towards framing SlowBreath as an aid for meditation. She would have preferred if this activity was framed as resting: "I react to the use of the word meditation. When I think about meditation, I think more about the east and finding Zen and such stuff, which does not suit me. So, for me, it is more a relaxation aid than a meditation aid".

\subsubsection{The Re-design of SlowBreath}

The participants overall liked the simplicity and the minimal interactions (choice of vibration and level of heat). They liked being able to remove all lights, close their eyes, lay in a comfortable position, and wear custom molded earplugs that removed all sound while using meditation devices, in order to minimize sensory engagements. When they were asked to compare SlowBreath to a range of existing solutions with audio or visual feedback, as described in Section 2.2, all participants said that the haptic feedback is strongly preferred to any other type of feedback. 
Participants expressed the desire for more control over parameters (vibration modes and heat in more granular increments), as represented by what Elisabeth said: "I would have liked to be able to change the length of the vibrational interval to six seconds instead of four. While using SlowBreath, I experienced that my breathing became so calm that longer intervals would be better. A button to adjust the strength and the depth of the vibration would be nice to have, so that one could do the adjustments with the eyes closed". Mathea, Henrik, and Astrid also pointed out that their reaction to haptic feedback depended on the state of the illness and the intensity of symptoms. For that too, adjustments would be important. Mathea expressed this common opinion as follows: "I have noticed that regarding the vibrations, I sometimes thought that it was a little too strong, and sometimes that it was a little too weak. So, it would have been nice if I could adjust the strength of the vibrations".

In addition to control over the parameters mentioned above, the participants would have appreciated having a timer. As it was, when using SlowBreath all participants set the alarm for 20-30 minutes. Mathea suggested the timer to have at least to options, a short 10-minute session, and a longer, 20 minutes one. The short option was perceived as a low threshold that was actually achievable for all.

Astrid and Mathea proposed an alternate placement of the vibration discs and the heat pads. Elisabeth explained: "I liked the feeling of the heat very much. So, I would always place the heat down on my stomach, and then, I would have wanted to have the vibration into my body, too. With SlowBreath as is, when the heat is placed down, the vibration side faced up. I had to use my hands to feel the vibration, but the hands are often something you should mentally disconnect from during meditation".

Inspired by thinking of how SlowBreath could help him practice energy balancing outside the home, Henrik came with the following suggestion: "I would have liked the cushion shaped like a fish. With a size that fits perfectly into my hands. It would be nice with a silicone surface, or something else that is easy to clean. I would then not have to worry about bringing home the bacteria from outside. If some kind of transparent silicone was used, then one could also choose whether you wanted vibration or a dimmed light to guide the breathing. The light alternative is suitable because vibration sometimes becomes a bit too disruptive. But also, the vibration feels really good. I could then just sit and have the fish inside my hand and use the vibration to calm down".

Other comments on the shape included: "The biggest round cushion gives the feeling of a real cushion, which for me resonates with an object made for a different use. If it was even slightly bigger, it could be seen as a sofa pillow, and as such, be very accessible. That is positive. However, people have different styles they prefer regarding their pillows", Elisabeth said. A re-design into a shape that fitted commercial sized cushion covers was perceived as a good option for Hedda: "It would be good if you can use your own cushion covers, then you have all the possibilities, and the style of the meditation cushion could be guided by a personal taste. It would be easily accessible since it is just there, on your couch. Nice with something you do not have to use the energy to get, or tidy after the use". As a possible negative aspect of this, she reflected, was that it could be easily forgotten - while in plain sight. Discussing the option of making the meditation cushion in teddy-bear shape, Hedda said: "An advantage with the teddy-bear shape is the possibility to personalize it. It could be given a name, and one could say 'I have to go to Teddy now, and rest a bit with him, coming back soon'”. The personalization could be a fun thing to do that could make it easier and perhaps more socially acceptable to take a break when in the company with others. Elin reflects along these lines: "In social situations, it can be very difficult to say that one needs a break. For example, if I am caught in an intense personal conversation, it can feel rude to break out of it. But the breaks for us with ME are necessary, or else one can easily sit for too long, risking becoming sicker. There is not a good way to tell others that you are in a bad shape".

All participants said that the aesthetics of the cushion was very important. As they spend a lot of time at home, every detail is extra important. Hedda articulated this point as follows: "Whether it is a cushion or a bear, I would have appreciated if it was designed with simplicity, and perceived as pretty. Then it is not an ugly thing that is at odds with the rest of the apartment when you bring it home". 


\section{DISCUSSION}

In this section, the three main implications of this work for designing technology for self-management of $\mathrm{ME}$, and for further research within HCI are discussed: 1) SlowBreath as part of energy balancing, 2) implications for design for relaxation for users with ME, and 3) The research approach.

\subsection{SlowBreath as Part of Energy Balancing}

The results of the study confirmed that people with ME are a user group with very different needs regarding the design of digital artefacts, including meditation cushions such as SlowBreath. The participants' condition demands minimal engagement and minimal interactions, in line with findings in (Culén et al., 2019). Working with them clearly demonstrated that over-burdening any of the five senses is not beneficial, and can trigger the hypersensitivity to external sensory inputs. The haptic feedback was found to be suitable, and in combination with heat, it was particularly helpful for people with ME, who often experience symptoms connected to abnormalities in the body temperature regulation (Carruthers et al., 2011). The findings indicate that self-management of ME through energy balancing can be helped with SlowBreath.

The findings also show that further improvements are necessary should SlowBreath be developed into a product. Most notably, the ability to easily (preferably with closed eyes) regulate the strength of the heath and vibration, the length of the vibrational cycle, as well as the length of a session would all be desirable features towards effective energy balancing.

\subsection{Meditation and ME, Implications for Design}

One of the insights was that, despite the small number of participants, the stand on meditation and ME reflected the pattern of the larger ME community - some participants were considering meditation as beneficial from the start, while others had reservations, and preferred to avoid the use of the word meditation all together (opting for describing the activities involved as the rest or relaxation). Apart from the terminology, the participants agree that the following implications regarding design for relaxation are important:

- The artefact for rest or meditation has to be carefully matched with their actual lives and needs that vary from day to day.

- The vibration and heat have to be adjustable and changeable during the experience of resting or meditating.

- A careful inquiry into how to support the development of sustained, everyday practices of using the artefact for meditation should be conducted, also from the perspective of those who do not like to call this practice meditation.

- In the same way as the computational materials need to be adjustable and controllable by participants, its shape and material components (fabrics) should match the style of the surroundings and hold whatever aesthetic standards the participant demands. Seeing how the actual home interior differed profoundly between participants, yet they all wanted the cushion to match their own interior, the ability to personalize the materials is particularly important when designing everyday artefacts for people with ME.

- The accessibility of the artefact within the home, is another important aspect when designing meditation artefacts for people with ME. If the artefact is an integral part of the home décor, and does not exert extra efforts to take it out, use it, or tidy after use, if it can facilitate resting at any time without taking extra energy (implying that it is accessible wherever rest and meditation take place), then it is beneficial for users with $\mathrm{ME}$, and lowers the threshold for use.

- Reminders to use the artefact should be considered when designing meditation artefacts for people with ME. Forgetting to take breaks is one of the main barriers to energy balancing. Setting up mobile phone reminders is a strategy that the participants already rely upon in their everyday lives, to overcome the cognitive symptoms associated with ME (challenges with memory and concentration). Thus, providing reminders is an important step towards facilitating the habitual use of SlowBreath as a self-management strategy for energy balancing. 


\subsection{The Research Approach}

Even though explorations were initially done by the author as a first-person research (Lucero et al., 2018), it was essential to deploy the SlowBreath in the field and with real users to get a proper indication of how well this product would work for people with ME. The insight from this study is the same as described in (Culén et al., 2019) - the research products placed in everyday lives offered the possibility for participants to use the research product as if it was a finished product, and to experience the artifact on their own terms. Of particular importance was that the use does not trigger the PEM. Furthermore, doing research with SlowBreath placed at people's homes gave the possibility to explore the bodily experiences of people with $\mathrm{ME}$ in a different way than traditional participatory approaches (where the design process is a co-design, but the artefacts are not always ready to be used at people's homes, in contrast to first-person design and lived experiences by users).

\subsection{Study Limitations}

The length of the study presented a limitation. Seeing how long it could take to develop the skill of meditation though mindfulness breathing and to develop and sustain practices of using SlowBreath as part of energy balancing, a lot longer study would have been needed. For in-depth insights on practices of meditation using SlowBreath by novice meditators and the potential health benefits would require a different time scale.

Another limitation is the small number of participants. This is frequently the case for similar qualitative studies in HCI, especially when the research involves vulnerable users.

Last, the interview data was coded and analyzed by the author only. To increase the validity of the analysis, as well as discuss the relevance of a-priory categories chosen, a collaboration with another researcher would be in place. However, SlowBreath is the last of several technology design explorations for people with ME done by the author during the last years. Thus, the author has relied on the extensive experience in the research domain that became the foundation for the approach taken.

\section{CONCLUSION}

This paper presented the design and deployment of a device called SlowBreath. SlowBreath was made with the intent to support people with $\mathrm{ME}$ in self-management through energy balancing. Unlike existing meditation aids that employ combined elements of audio, haptic and visual feedback to guide the user in achieving a meditative state, SlowBreath takes the alternate approach relying on minimal interactions and haptic feedback through vibration and heat to avoid PEM due to sensory overload.

Overall, all five participants who took part in the study preferred the haptic feedback over the other devices available that used combinations of haptic, audio and visual feedback. The findings also highlight the importance of exploring the combinations and dynamics of the materials, form and computational expressions when designing for technologically-mediated meditative experiences.

\section{ACKNOWLEDGEMENT}

Thanks are due to the Norwegian ME organization for helping with the participant recruitment process, and providing helpful advice anytime it was needed. I would also like to thank the participants who generously shared their time, and provided valuable input and feedback on their experiences of having SlowBreath in their lives for the purpose of this study.

\section{REFERENCES}

Bergström, I., Jonsson, M., 2016. Sarka: Sonification and Somaesthetic Appreciation Design. Proceedings of the 3rd International Symposium on Movement and Computing - MOCO '16. Thessaloniki, GA, Greece, pp. 1-8.

Brown, K. W., Creswell, J. D., Ryan, R. M., 2015. Handbook of mindfulness: theory, research, and practice. The Guilford Press, New York. 
Calvo, R. A., Peters, D., 2014. Positive computing: technology for wellbeing and human potential. The platform studies series. The MIT Press, Cambridge, Massachusetts, USA.

Carruthers, B. M., van de Sande, M. I., De Meirleir, K. L., Klimas, N. G., Broderick, G., Mitchell, T., et al., 2011. Myalgic encephalomyelitis: International Consensus Criteria. Journal of internal medicine, Vol. 270, No. 4, pp. 327-338.

Cochrane, K. A., Loke, L., de Bérigny, C., Campbell, A., 2018. Sounds in the moment: designing an interactive EEG nature soundscape for novice mindfulness meditators. Proceedings of the 30th Australian Conference on Computer-Human Interaction - OzCHI '18. Melbourne, Australia, pp. 298-302.

Culén, A. L., Børsting, J., Odom, W., 2019. Mediating Relatedness for Adolescents with ME: Reducing Isolation through Minimal Interactions with a Robot Avatar. Proceedings of the 2019 on Designing Interactive Systems Conference 2019 - DIS '19, ACM, San Diego, USA. Doi:10.1145/3322276.3322319

Goudsmit, E. M., Nijs, J., Jason, L. A., Wallman, K. E., 2012. Pacing as a strategy to improve energy management in myalgic encephalomyelitis/chronic fatigue syndrome: a consensus document. Disability and rehabilitation, Vol. 34, No. 13, pp. 1140-1147.

Hvidberg, M. F., Brinth, L. S., Olesen, A. V., Petersen, K. D., Ehlers, L., 2015. The Health-Related Quality of Life for Patients with Myalgic Encephalomyelitis/Chronic Fatigue Syndrome (ME/CFS). PLOS ONE, Vol. 10, No. 7, pp.1-16.

Höök, K., Jonsson, M. P., Ståhl, A., Mercurio, J., 2016. Somaesthetic Appreciation Design. Proceedings of the 2016 CHI Conference on Human Factors in Computing Systems - CHI '16. ACM Press, California, USA, pp. 3131-3142.

Höök, K., 2018. Designing with the body: somaesthetic interaction design. The MIT Press, Cambridge, Massachusetts, USA.

Janlert, L.-E., Stolterman, E., 2017. The Meaning of Interactivity-Some Proposals for Definitions and Measures. Human-Computer Interaction, Vol. 32, No. 3, pp. 103-138.

Kabat-Zinn, J., 1990. Full catastrophe living: using the wisdom of your body and mind to face stress, pain, and illness. Delacorte Press, New York, NY. USA.

Kaye, J. J., 2006. I Just Clicked to Say I Love You: Rich Evaluations of Minimal Communication. Extended Abstracts on Human Factors in Computing Systems CHI'06. New York, NY, USA, pp. 363-368.

Lacourt, T. E., Vichaya, E. G., Chiu, G. S., Dantzer, R., Heijnen, C. J., 2018. The High Costs of Low-Grade Inflammation: Persistent Fatigue as a Consequence of Reduced Cellular-Energy Availability and Non-adaptive Energy Expenditure. Frontiers in behavioral neuroscience, Vol. 12, No. 78, pp. 1-20.

Lazar, J., Feng, J. H., Hochheiser, H., 2010. Research methods in human computer interaction, John Wiley \& Sons, NJ, USA.

Líndal, E., Stefánsson, J. G., Bergmann, S., 2002. The prevalence of chronic fatigue syndrome in Iceland - A national comparison by gender drawing on four different criteria. Nordic journal of psychiatry, Vol. 56, No. 4, pp. 273-277.

Lucero, A., Desjardins, A., Neustaedter, C., Höök, K., Hassenzahl, M., Cecchinato, M.E., 2018. A Sample of One: First-Person Research Methods in HCI, Proceedings of the 2019 ACM Conference Companion Publication on Designing Interactive Systems. ACM.

Mazé, R., Redström, J., 2005. Form and the computational object. Digital Creativity. Vol. 16, No. 1, pp. 7-18.

Niksirat, K. S., Silpasuwanchai, C., Ahmed, M. M. H., Cheng, P., Ren, X., 2017. A Framework for Interactive Mindfulness Meditation Using Attention-Regulation Process. Proceedings of the 2017 CHI Conference on Human Factors in Computing Systems - CHI '17. Denver, Colorado, USA, pp. 2672-2684.

Odom, W., Wakkary, R., Lim, Y., Desjardins, A., Hengeveld, B., Banks, R., 2016. From Research Prototype to Research Product. Proceedings of the 2016 CHI Conference on Human Factors in Computing Systems, CHI '16. ACM. New York, NY, USA, pp. 2549-2561.

Prpa, M., Tatar, K., Françoise, J., Riecke, B., Schiphorst, T., Pasquier, P., 2018. Attending to Breath: Exploring How the Cues in a Virtual Environment Guide the Attention to Breath and Shape the Quality of Experience to Support Mindfulness. Proceedings of the 2018 on Designing Interactive Systems Conference 2018 - DIS '18. Hong Kong, China, pp. 71-84.

Sanches, P., Höök, K., Vaara, E., Weymann, C., Bylund, M., Ferreira, P., et al., 2010. Mind the body!: designing a mobile stress management application encouraging personal reflection. Proceedings of the 8th ACM Conference on Designing Interactive Systems - DIS '10. Aarhus, Denmark, pp. 47-56.

Shusterman, R., 2012. Thinking through the Body: Essays in Somaesthetics. Cambridge University Press, Cambridge, United Kingdom.

Ståhl, A., Jonsson, M., Mercurio, J., Karlsson, A., Höök, K., Johnson, E.-C. B., 2016. The Soma Mat and Breathing Light. Proceedings of the 2016 CHI Conference Extended Abstracts on Human Factors in Computing Systems - CHI EA '16, ACM Press, Santa Clara, California, USA, pp. 305-308. 